\title{
Multi-scale modeling of moving interface problems with flux and field jumps: Application to oxidative degradation of ceramic matrix composites
}

\author{
Sangmin Lee and Veera Sundararaghavan $*, \dagger, \dot{\dagger}$ \\ Department of Aerospace Engineering, University of Michigan, Ann Arbor, MI 48109, U.S.A.
}

\begin{abstract}
SUMMARY
Problems involving reaction and species diffusion involve field and flux jumps at a moving reaction front. In multi-scale problems such as carbon fiber composite oxidation, these effects need to be tracked at the microscopic scale of individual carbon fibers. A multi-scale model is derived in this paper for predicting species distribution in such problems using a fully coupled multi-scale homogenization approach. The homogenized fluxes from the micro-scale are derived using Hill's macro-homogeneity condition accounting for both flux jumps and species density field jumps at the reacting interface in the micro-scale unit cell. At the macro-scale, the competition between the transport of reacting species (oxygen) and the reaction product (carbon dioxide) is modeled using homogenized mass conservation equations. The moving reaction front in carbon fibers at the micro-scale is tracked using level set method and an adaptive meshing strategy. The macroscopic weight loss of the composite when exposed to oxygen is simulated as a function of time using a coupled finite element methodology at various locations in a validated macroscopic model. Copyright (C) 2010 John Wiley \& Sons, Ltd.
\end{abstract}

Received 4 September 2009; Revised 10 June 2010; Accepted 15 June 2010

KEY WORDS: multi-scale modeling; interface evolution; oxidation; carbon composites; homogenization

\section{INTRODUCTION}

Advanced launch vehicles employ ceramic matrix composites (CMCs) as structural elements in heat shields, propulsion components and other applications that encounter high temperature oxidizing environments. These conditions progressively degrade the material that eventually leads to failure of the component well short of its expected life [1]. A variety of degradation mechanisms exist (Figure 1), with the predominant one being the oxidation of $\mathrm{C}$ fibers. For moderate to high temperatures, this oxidation process is controlled by the transport of oxygen into the porous $\mathrm{SiC}$ matrix. The oxygen transport phenomena in $\mathrm{C} / \mathrm{SiC}$ composites is primarily through an interconnected void network in the matrix formed due to thermal expansion mismatch between carbon fibers and the matrix during processing. At the micro-scale, fine carbon fibers in within tows interact with oxygen and degrade at moderate to high temperatures. Initial models for studying oxidation of $\mathrm{C} / \mathrm{SiC}$ composites at micro-scales assumed a steady-state diffusion through the matrix $[2,3]$. While these techniques model the micro-scale, techniques for homogenizing the results for use in global analysis was not developed. In [4], a continuum-level theory for modeling composite oxidation was

\footnotetext{
${ }^{*}$ Correspondence to: Veera Sundararaghavan, Department of Aerospace Engineering, University of Michigan, Ann Arbor, MI 48109, U.S.A.

†E-mail: veeras@umich.edu

¥Assistant Professor.
} 


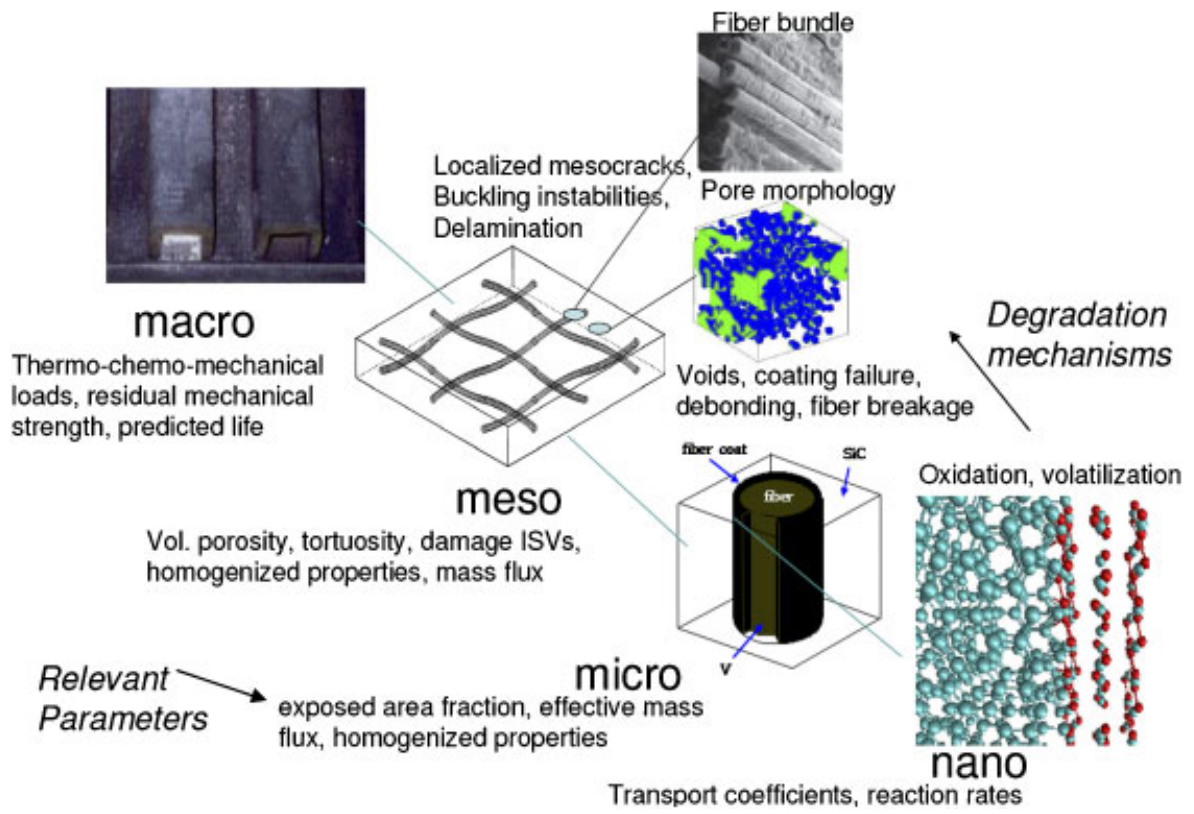

Figure 1. $\mathrm{C} / \mathrm{SiC}$ composites in extreme thermo-chemo-mechanical loading conditions are subject to a variety of degradation mechanisms. Modeling of macro-scale degradation should involve physical models at multiple length scales. In this paper, we focus on micro-scale degradation modeling using finite element analysis.

developed. The approach models the composite as a homogeneous mixture of matrix, carbon fiber and voids. While continuum models are practical approaches for predicting the behavior of $\mathrm{C} / \mathrm{SiC}$ composites, they do not model the inherent heterogeneities involved in fiber oxidation. Multi-scale modeling by coupling macroscopic and microscopic models allows us to take advantage of both the efficiency of continuum models and the accuracy of the microscopic models.

Multi-scale analysis of diffusion problems have been previously addressed using micro-scale effective properties obtained through either bounding relations $[5,6]$ or analytical closed-form expressions (reviewed in [7]) in a macro-scale model. These approaches were restricted to simple geometries with a simple material response, not valid when degrading interfaces are present. More recently, numerical schemes using asymptotic homogenization approaches, based on an expansion of the unknown field variable with respect to a micro-scale length parameter, have been developed to address micro-macro diffusion problems [8-10]. However, the problems considered are restricted to constant transport properties and focused on steady-state diffusion problems. Oxidation problems involve transient effects, and in addition, involve interface phenomena that have not been previously addressed in a multi-scale methodology. Computational homogenization provides an attractive avenue for computing the macroscopic response in problems with discontinuities and non-linearities. Computational homogenization is a multi-scale analysis approach in which computations are concurrently performed at two different length-scales. The macro-scale is associated with the component being modeled $\left(10^{-3}\right.$ to $\left.10^{+1} \mathrm{~m}\right)$ and the meso-scale is characterized by the underlying composite microstructure $\left(10^{-6}\right.$ to $\left.10^{-3} \mathrm{~m}\right)$. The principle of scale separation states that the characteristic length scale over which the macroscopic field variables vary should be much larger than the size of the microscopic volume considered. In other words, macroscopic quantities are nearly constant at the level of a representative volume element (RVE). An RVE is defined at the micro-scale and boundary conditions are defined on the RVE in terms of macroscopic quantities. Applications of such approaches for thermo-mechanical deformation have been well studied previously [11-13].

Recently, Belytschko et al. [14] have modeled material discontinuities (cracks) across lengthscales in solids using this technique. Complementing this development, we focus on problems 
involving field and flux discontinuities. Flux discontinuities arise in several problems, the most well studied of which is fluid solidification. In solidification problems, a flux jump (manifested as latent heat) occurs at the evolving solidification front whereas the overall temperature field itself is continuous in the domain. Recently, the homogenization scheme was extended for addressing such problems in our recent work [15]. Oxidation problem addressed in this work not only involves a flux discontinuity but also additionally involves a field discontinuity in the form of a jump in oxygen density field across the oxidizing interface. In this paper, we develop a fully coupled multi-scale homogenization approach for problems involving both field and flux discontinuities. The paper is organized as follows: In Sections 2 and 3, a generalized scale transition for the multiscale problem is derived. The micro-scale and the macro-scale models are explained in Sections 4 and 5, respectively. In Section 6, the level set method used to model the moving interface is explained. Finally, in Section 7, we discuss the methodology through an example of oxidation of a carbon composite.

\section{COMPUTATIONAL HOMOGENIZATION APPROACH: MICRO-SCALE BOUNDARY CONDITIONS}

We consider a problem of oxidation, where carbon fibers residing in an inert matrix reacts with oxygen. The microstructure of a 2-D C/SiC composite is shown in Figure 2. The unit cell consists of a single carbon fiber in a porous matrix occupying a volume $V$ with external boundary $S$ and outward normal $\boldsymbol{n}$. The oxidizing interface is a moving internal boundary, which is initially an intact fiber-matrix interface. Material outside of the exposed carbon fiber surface occupies a volume $V^{+}$and the exposed carbon fiber occupies a volume $V^{-}$. Here, $V^{+}$and $V^{-}$are open subsets of the total unit cell volume $V$. At the interface $S^{\mathrm{I}}$ between $V^{+}$and $V^{-}$, the carbon fiber oxidizes further and recedes into $V^{-}$along $\boldsymbol{n}^{\mathrm{I}}$ with speed $v_{n}$, where $\boldsymbol{n}^{\mathrm{I}}$ is the inward normal of $S^{\mathrm{I}}$ as indicated in Figure 2. One such unit cell is attached to every integration point in the macro-scale finite element mesh.

To identify boundary conditions that need to be employed at the unit-cell level, we employ the computational homogenization approach developed in $[11-13,16]$. Macro-micro linking is achieved by decomposing the micro-scale field $(\rho)$ into a sum of macroscopic field and a fluctuation field $(\tilde{\rho})$ as:

$$
\rho=\rho_{\text {ref }}+\overline{\nabla \rho} \cdot \boldsymbol{x}+\tilde{\rho}
$$

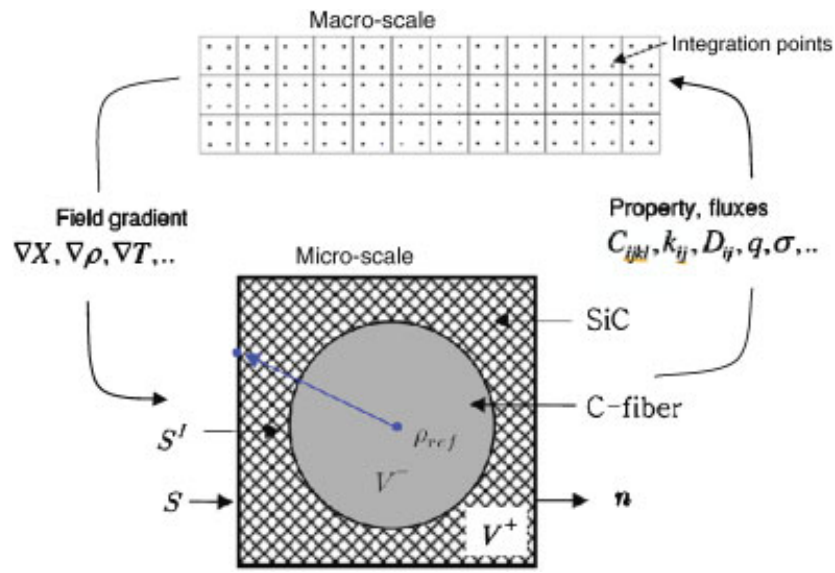

Figure 2. Macro-scale is associated with a homogenized continuum. The macro-scale fields and field gradients are passed to the micro-scale as boundary conditions. Macro-scale fluxes and properties (at all integration points) are computed from the underlying microstructural sub-problems using averaging schemes. 
Here, the coordinate $\boldsymbol{x}$ represents a point on the micro-scale relative to a reference point at the center of the unit cell. The micro-scale partial density field of species $i$ is denoted as $\rho$ and the macroscopic reference partial density field of species $i$ is denoted as $\rho_{\text {ref. No subscripts (for }}$ eg. $\rho_{i}$ for species $i$ ) are used in this section to maintain generality.

In general, we denote a macroscopic counterpart of a microscopic quantity (say, $\chi$ ) as $\bar{\chi}$. In the above equation, gradient in partial density of specie $i$ at the macroscopic material point is denoted as $\overline{\nabla \rho}\left(=\nabla_{\text {macro }} \bar{\rho}\right)$. The most general assumption behind homogenization theory is that the gradient as seen at the macro-scale $(\overline{\nabla \rho})$ can be represented purely in terms of the field variables at the exterior boundary of the microstructure $[15,17]$ :

$$
\overline{\nabla \rho}=\frac{1}{V} \int_{S} \rho \boldsymbol{n} \mathrm{d} S
$$

Using the micro-scale field decomposition (Equation (1)), it can be shown that:

$$
\frac{1}{V} \int_{V} \nabla \rho \mathrm{d} V=\bar{\nabla} \rho+\frac{1}{V} \int_{V} \nabla \tilde{\rho} \mathrm{d} V
$$

We employ the generalized divergence theorem of the form $\int_{V} \nabla \chi \mathrm{d} V=\int_{S} \chi \boldsymbol{n} \mathrm{d} S+\int_{S^{\mathrm{I}}}[|\chi|] \boldsymbol{n}^{\mathrm{I}} \mathrm{d} S^{\mathrm{I}}$ in the above equation (where $[|\chi|]$ denotes the jump in the field quantity across the evolving interface) to obtain the following relationship:

$$
\frac{1}{V} \int_{S} \rho \boldsymbol{n} \mathrm{d} S=\bar{\nabla} \rho+\frac{1}{V} \int_{S} \tilde{\rho} \boldsymbol{n} \mathrm{d} S+\frac{1}{V} \int_{S^{\mathrm{I}}}([|\tilde{\rho}|]-[|\rho|]) \boldsymbol{n}^{\mathrm{I}} \mathrm{d} S^{\mathrm{I}}
$$

In the above equation, $[|\rho|]$ denotes the jump in partial density of specie $i$ across the interface $\left(S^{\mathrm{I}}\right)$ with normal $n^{\mathrm{I}}$. The jump in field across an interface is computed as $[|\rho|]=\rho_{+}-\rho_{-}$. Here, $\rho_{+}$and $\rho_{-}$refer to the field values in domain $V^{+}$and $V^{-}$, respectively, close to a point on the interface.

Boundary conditions at the micro-scale are identified by comparing Equation (2) with (4). Thus, any such boundary conditions derived must satisfy the 'linking condition':

$$
\frac{1}{V} \int_{S} \tilde{\rho} \boldsymbol{n} \mathrm{d} S+\frac{1}{V} \int_{S^{\mathrm{I}}}([|\tilde{\rho}|]-[|\rho|]) \boldsymbol{n}^{\mathrm{I}} \mathrm{d} S^{\mathrm{I}}=0
$$

A variety of boundary conditions may be derived that satisfy this constraint, however, following our previous work in [15], we focus on two possible boundary conditions (also called 'macro-micro linking assumption') as given below:

(1) Taylor boundary condition involves specification of density field at all points in the microstructure. The approach is called 'Taylor model' based on similar terminology used in multi-scale deformation problems where displacements are fully specified at micro-scale. The Taylor model involves the following boundary conditions:

$$
\begin{array}{ll}
\tilde{\rho}=0 & \text { on } V^{+} \\
\rho=0 & \text { on } V^{-}
\end{array}
$$

The first term in Equation (5) vanishes based on the first condition (Equation (6)). The second equation implies that the density of oxygen (and carbon dioxide) is negligible inside the carbon fiber. The mechanism of transport of oxygen in the carbon fiber is through lattice diffusion and this rate of diffusion is indeed negligible compared with gas phase transport (within pores) as noted in diffusion studies [18]. Comparing Equation (7) with (1) leads to expression for $\tilde{\rho}$ inside the carbon fiber: $\tilde{\rho}=-\rho_{\text {ref }}-\overline{\nabla \rho} \cdot \boldsymbol{x}$ on $V^{-}$. This leads to the expression for jump in the fluctuation density field at the interface:

$$
[|\tilde{\rho}|]=\rho_{\text {ref }}+\overline{\nabla \rho} \cdot \boldsymbol{x} \quad \text { on } S^{\mathrm{I}}
$$

Similarly, comparing Equation (6) with (1) leads to $\rho=\rho_{\text {ref }}+\overline{\nabla \rho} \cdot \boldsymbol{x}$ on $V^{+}$. Since $\rho=0$ on $V^{-}$(Equation (7)), we can obtain the expression for jump in field at the interface

$$
[|\rho|]=\rho_{\text {ref }}+\overline{\nabla \rho} \cdot \boldsymbol{x} \quad \text { on } S^{\mathrm{I}}
$$


From Equations (8) and (9), it can be verified that the expression $[|\tilde{\rho}|]-[|\rho|]$ in Equation (5) also vanishes at points on the interface $S^{\mathrm{I}}$. Thus, the Taylor assumption fully satisfies the linking condition (Equation (5)). Note that when using the Taylor linking assumption, the density fields at the micro-scale are fully specified from macro-scale quantities using the following equation:

$$
\begin{aligned}
& \rho=\rho_{\text {ref }}+\overline{\nabla \rho} \cdot x \text { on } V^{+} \\
& \rho=0 \quad \text { on } V^{-}
\end{aligned}
$$

(2) Homogenization boundary condition: The essential boundary condition of the following form is specified:

$$
\begin{array}{rlrl}
\tilde{\rho} & =0 & & \text { on } S \\
([|\tilde{\rho}|]-[|\rho|])=0 & & \text { on } S^{\mathrm{I}}
\end{array}
$$

Using Equation (11), essential boundary conditions (in the form of densities of oxygen and oxide species) are enforced at the boundaries of the unit cell as:

$$
\rho=\rho_{\text {ref }}+\overline{\nabla \rho} \cdot \boldsymbol{x} \quad \text { on } S
$$

The second constraint in Equation (12) is trivially satisfied based on Equation (1) since macroscopic fields are assumed to be continuous. In homogenization approach, the density fields are known at the external boundary and micro-scale equilibrium equations are solved using finite element analysis to completely describe the oxygen density distribution at the micro-scale.

\section{MICRO-MACRO LINKING: TRANSFERRING FLUXES AND DIFFUSIVITIES}

In a coupled multi-scale model, the micro-scale results are used to compute transport properties and mass flux of species at the macro-scale. This forms the basis for micro-to-macro linking. In particular, we are interested in obtaining a macroscopic flux that satisfies Hills macro-homogeneity condition (which relates the macroscopic flux $(\overline{\boldsymbol{q}})$ with its microstructural counterpart $(\boldsymbol{q}[15,19])$ as follows:

$$
\overline{\nabla \rho} \cdot \overline{\boldsymbol{q}}=\overline{\nabla \rho \cdot \boldsymbol{q}}
$$

The fluxes need to be derived such that the above macro-homogeneity condition is satisfied when using either Taylor or homogenization boundary conditions as follows:

- The Taylor model. Using boundary conditions (Equations (6) and (7)) in the macrohomogeneity condition (Equation (14)) leads to the following equation:

$$
\begin{aligned}
\overline{\nabla \rho \cdot \boldsymbol{q}}=\frac{1}{V} \int_{V} \nabla \rho \cdot \boldsymbol{q} \mathrm{d} V & =\frac{1}{V} \int_{V} \nabla\left(\rho_{\mathrm{ref}}+\overline{\nabla \rho} \cdot \boldsymbol{x}\right) \cdot \boldsymbol{q} \mathrm{d} V \\
& =\overline{\nabla \rho} \cdot \frac{1}{V} \int_{V} \nabla \boldsymbol{x} \cdot \boldsymbol{q} \mathrm{d} V=\bar{\nabla} \rho \cdot \frac{1}{V} \int_{V} \boldsymbol{q} \mathrm{d} V
\end{aligned}
$$

Comparing Equation (15) with (14):

$$
\overline{\boldsymbol{q}}=\frac{1}{V} \int_{V} \boldsymbol{q} \mathrm{d} V
$$

- The Homogenization model. We can show that a similar expression holds for the homogenization approach, ie. the macroscopic flux is same as the volume-averaged heat flux at the micro-scale as shown in the following equation:

$$
\overline{\boldsymbol{q}}=\frac{1}{V} \int_{S} \boldsymbol{x} q_{n} \mathrm{~d} S=\frac{1}{V} \int \boldsymbol{q} \mathrm{d} V
$$


Derivation of the macroscopic flux for homogenization approach is more detailed than the derivation shown for the Taylor model. The derivation, which is based on the model explained in Section 4, is separately provided in Appendix A for the interested reader.

\section{MICRO-SCALE MODEL}

Since the microscopic length scale is considered to be much smaller than the scale of variation of the macroscopic temperature field, the micro-scale can be assumed to be at steady state at any instant of the macroscopic (transient) evaluation. This is a general assumption in homogenization theory due to the scale separation principle (e.g. [16]).

The microscopic mass conservation equation is given as

$$
\nabla \cdot \boldsymbol{q}_{i}=-\nabla \cdot\left(\rho_{i} \boldsymbol{v}\right)
$$

where $\boldsymbol{q}_{i}$ denotes the mass flux of species $i$ and $\rho_{i}$ denotes the partial density of species $i$, and $v$ represents the velocity of recession of the exposed carbon ( $v$ is non-zero only on the evolving interface). The micro-scale model includes both density jump ([|$\left.\left.\rho_{i} \mid\right]\right)$ and flux jump $\left(\left[\left|\boldsymbol{q}_{i}\right|\right]\right)$ for each specie $i$ in the normal direction across the oxidizing interface. The condition for local mass balance across the oxidizing interface (with the flux and velocity component along the normal to the interface given as $q_{n}$ and $v_{n}$, respectively) is given by the local form of the Rankine-Hugoniot condition $[20,21]$ :

$$
\left(\left[\left|q_{n_{i}}\right|\right]+v_{n}\left[\left|\rho_{i}\right|\right]\right)=0
$$

As described before, the primary mechanism of oxygen transport is concentration and pressure gradient-driven diffusion through the pores (or cracks) in the matrix. In comparison, the species density and mass flux inside the carbon fiber is negligible (ie. $\boldsymbol{q}_{i}^{-}=\mathbf{0}$ ). Thus, the following interface flux condition can be derived to simulate mass loss of carbon fiber during oxidation:

$$
\boldsymbol{q}_{\mathrm{O}_{2}}^{+}=-\frac{M_{\mathrm{O}_{2}}}{M_{\mathrm{C}}} \rho_{\mathrm{c}} \boldsymbol{v}, \quad \boldsymbol{q}_{\mathrm{CO}_{2}}^{+}=\frac{M_{\mathrm{CO}_{2}}}{M_{\mathrm{C}}} \rho_{\mathrm{c}} \boldsymbol{v} \quad \text { on } S^{\mathrm{I}}
$$

where $\rho_{\mathrm{c}}$ is the density of the carbon fiber and $M_{i}$ denotes the molecular mass of species $i$. The sign in the above equation indicates that oxygen is consumed and carbon dioxide is released at the interface during oxidation. The presence of interface flux at the micro-scale leads to a homogenized source term (for oxygen and carbon dioxide densities) in the macroscopic model.

\subsection{Description of flow in the porous matrix at micro-scale}

The mass flux of species within the matrix with volumetric porosity $(\phi)$ is a result of both pressure gradient-driven flow and concentration gradient-driven flow. The net flux is represented as

$$
\boldsymbol{q}=\boldsymbol{q}_{i}^{\alpha}+\boldsymbol{q}_{i}^{\beta}
$$

where $\boldsymbol{q}_{i}^{\alpha}$ and $\boldsymbol{q}_{i}^{\beta}$ are the mass flux of species $i$ due to pressure gradient-driven flow and concentration gradient-driven flow, respectively.

The flux contribution within the porous matrix due to pressure gradient-driven flow is given by the Darcy equation as:

$$
\boldsymbol{q}_{i}^{\alpha}=-\rho_{i}^{\mathrm{p}} \frac{1}{\mu_{\mathrm{g}}} \mathbf{k} \nabla P
$$

Here, $\rho_{i}^{\mathrm{p}}=\rho_{i} / \phi$ denotes the partial density of the species $i$ in the pore. In addition, $\mu_{\mathrm{g}}$ denotes the viscosity of the gas mixture, $\mathbf{k}=k \boldsymbol{I}$ ( $\boldsymbol{I}$ is the identity matrix) denotes the second-order material permeability tensor and $P\left(=\sum_{i} P_{i}=P_{\mathrm{O}_{2}}+P_{\mathrm{CO}_{2}}\right)$ represents the total pressure of the gas mixture within the pore. The partial pressures $\left(P_{i}\right)$ for each specie $i$ are obtained using the ideal gas law 
( $\rho_{i}^{\mathrm{p}}=M_{i} P_{i} /(R T)$, where $R$ is the universal gas constant and $T$ is the temperature). Similarly, the density of the mixture within the pore can be computed as $\rho^{\mathrm{p}}=M P /(R T)$, where $M$ is the averaged molecular weight of the gas mixture.

The concentration gradient-driven mass flux in the porous matrix (based on Reference [4]) is given using Fick's law of diffusion that involves the second-order areal porosity tensor $\left(\varphi^{A}\right)$, which is used as a measure of resistance to concentration gradient-driven flow through the pore network:

$$
\boldsymbol{q}_{i}^{\beta}=-\rho^{\mathrm{p}} D \boldsymbol{\varphi}^{A} \nabla\left(\frac{\rho_{i}^{\mathrm{p}}}{\rho^{\mathrm{p}}}\right)
$$

where $D$ is the diffusivity of oxygen w.r.t. carbon dioxide. The areal porosity tensor can be thought of as a ratio of porosity to the tortuosity [22]. We employ the calibrated isotropic areal porosity from Reference [4] for the matrix in the micro-scale calculations. The diffusivity $D$ of gas specie A w.r.t. gas specie B is given by the Chapman-Enskog equation [23]:

$$
D=0.0018583 \frac{\sqrt{T^{3}\left(\frac{1}{M_{A}}+\frac{1}{M_{B}}\right)}}{P \sigma_{A B}^{2} \Omega_{D, A B}}\left[\mathrm{~cm}^{2} / \mathrm{s}\right]
$$

where $M_{i}$ is the molecular weight of species $i$ and $\Omega_{D, A B}$ and $\sigma_{A B}$ are computed from the Lennard-Jones potentials of the respective species (the parameters employed are tabulated in Table I).

Based on Equations (21)-(23), the constitutive relationships between homogenized fluxes and pressure gradients of oxygen and carbon dioxide can be derived as follows:

$$
\begin{gathered}
\boldsymbol{q}_{\mathrm{O}_{2}}=\boldsymbol{\kappa}^{\mathrm{O}_{2} / \mathrm{O}_{2}} \nabla \rho_{\mathrm{O}_{2}}+\boldsymbol{\kappa}^{\mathrm{O}_{2} / \mathrm{CO}_{2}} \nabla \rho_{\mathrm{CO}_{2}} \\
\boldsymbol{q}_{\mathrm{CO}_{2}}=\boldsymbol{\kappa}^{\mathrm{CO}_{2} / \mathrm{O}_{2}} \nabla \rho_{\mathrm{O}_{2}}+\boldsymbol{\kappa}^{\mathrm{CO}_{2} / \mathrm{CO}_{2}} \nabla \rho_{\mathrm{CO}_{2}}
\end{gathered}
$$

where $\boldsymbol{\kappa}^{A / B}$ denotes the micro-scale diffusivity relating the mass flux of species A with respect to the pressure gradient of species $B$ and is computed as follows:

$$
\begin{aligned}
\boldsymbol{\kappa}^{\mathrm{O}_{2} / \mathrm{O}_{2}} & =-P_{\mathrm{O}_{2}} \frac{1}{\phi \mu_{\mathrm{g}}} \mathbf{k}-\frac{D}{\phi} \boldsymbol{\varphi}^{A} \frac{P_{\mathrm{CO}_{2}}}{P} \\
\boldsymbol{\kappa}^{\mathrm{O}_{2} / \mathrm{CO}_{2}} & =\frac{M_{\mathrm{O}_{2}}}{M_{\mathrm{CO}_{2}}}\left(-P_{\mathrm{O}_{2}} \frac{1}{\phi \mu_{\mathrm{g}}} \mathbf{k}+\frac{D}{\phi} \boldsymbol{\varphi}^{A} \frac{P_{\mathrm{O}_{2}}}{P}\right) \\
\boldsymbol{\kappa}^{\mathrm{CO}_{2} / \mathrm{O}_{2}} & =\frac{M_{\mathrm{CO}_{2}}}{M_{\mathrm{O}_{2}}}\left(-P_{\mathrm{CO}_{2}} \frac{1}{\phi \mu_{\mathrm{g}}} \mathbf{k}+\frac{D}{\phi} \boldsymbol{\varphi}^{A} \frac{P_{\mathrm{CO}_{2}}}{P}\right) \\
\boldsymbol{\kappa}^{\mathrm{CO}_{2} / \mathrm{CO}_{2}} & =-P_{\mathrm{CO}_{2}} \frac{1}{\phi \mu_{\mathrm{g}}} \mathbf{k}-\frac{D}{\phi} \boldsymbol{\varphi}^{A} \frac{P_{\mathrm{O}_{2}}}{P}
\end{aligned}
$$

\begin{tabular}{|c|c|c|c|c|}
\hline & $\sigma(\AA)$ & $\frac{\varepsilon}{k}(K)$ & $\sigma_{A B}(\AA)$ & $\frac{\varepsilon_{A B}}{k}(K)$ \\
\hline $\begin{array}{l}\mathrm{O}_{2} \\
\mathrm{CO}_{2}\end{array}$ & $\begin{array}{l}3.433 \\
3.996\end{array}$ & $\begin{array}{l}113 \\
190\end{array}$ & $\begin{array}{l}=\frac{1}{2}\left(\sigma_{A}+\sigma_{B}\right) \\
=3.7145\end{array}$ & $\begin{array}{l}=\frac{\sqrt{\varepsilon^{\varepsilon^{\varepsilon} B}}}{k} \\
=146\end{array}$ \\
\hline Temp. $\left({ }^{\circ} \mathrm{C}\right)$ & & & & $\Omega_{D, A B}=f\left(\frac{k T}{\varepsilon_{A B}}\right)$ \\
\hline $\begin{array}{l}900 \\
950\end{array}$ & & & & $\begin{array}{l}0.7710 \\
0.7657\end{array}$ \\
\hline
\end{tabular}

Table I. Lennard Jones potential parameters for the diffusivity between $\mathrm{O}_{2}$ and $\mathrm{CO}_{2}$ ( $k$ : Boltzmann constant). 
The computational approach used to solve the micro-scale field and flux distribution using the Taylor and homogenization approaches are listed below:

(1) Micro-scale model-Taylor approach: The oxygen and carbon dioxide density field distributions in the micro-scale are directly obtained from the macro-scale solution using the Taylor boundary conditions (Equation (10)). The fluxes at all points within the microstructure are subsequently calculated using Equation (25). Note that the Taylor solution may not satisfy the micro-scale equilibrium (Equation (18)).

(2) Micro-scale model_Homogenization approach: Boundary conditions (Equation (13)) are specified in each unit cell. The two coupled non-linear partial differential equations (Equation (18)), one equation each for $\mathrm{O}_{2}$ and $\mathrm{CO}_{2}$ are solved using finite element analysis. To solve these non-linear equations, Galerkin finite element method is adopted and the weak form is solved in an incremental iterative manner using the Newton-Raphson method.

\section{MACRO-SCALE MODEL}

The macroscopic simulation is performed using a diffusion equation that is defined on a uniformly meshed domain $(\Omega)$ with boundaries defined as $\Gamma$. Degrading interfaces are explicitly modeled at the micro-scale, whereas only homogenized transport equations are modeled at the macro-scale as given as follows:

$$
\begin{aligned}
\frac{\partial \bar{\rho}_{\mathrm{O}_{2}}}{\partial t}+\nabla \cdot \overline{\boldsymbol{q}}_{\mathrm{O}_{2}} & =\bar{Q}_{\mathrm{O}_{2}} \\
\frac{\partial \bar{\rho}_{\mathrm{CO}_{2}}}{\partial t}+\nabla \cdot \overline{\boldsymbol{q}}_{\mathrm{CO}_{2}} & =-\bar{Q}_{\mathrm{CO}_{2}} \\
\bar{\rho}(\Gamma, t>0) & =\hat{\rho}, \quad \bar{\rho}(\Omega, t=0)=\bar{\rho}_{0}
\end{aligned}
$$

where $\bar{\rho}$ is the macroscopic (homogenized) partial density and $\bar{Q}_{i}$ is the homogenized mass consumption rate of species $i$ per unit bulk volume defined as (where $f$ is the volume fraction of the burnt carbon fiber at the micro-scale):

$$
\bar{Q}_{i}=\rho_{\mathrm{c}} \frac{M_{i}}{M_{\mathrm{c}}} \frac{\partial f}{\partial t}
$$

To solve the non-linear transient macroscopic equations (Equation (27)), the Galerkin finite element method and backward Euler time integration are adopted and the weak form is solved in an incremental iterative manner using the Newton-Raphson method. The $(\lambda+1)$ th Newton-Raphson step at time $(t+1)$ involves solution of the system $\boldsymbol{K}\left\{\delta \overline{\boldsymbol{\rho}}^{\lambda+1, t+1}\right\}=\boldsymbol{f}$, where the unknown vector in the above system is the increment in the partial density $\left(\delta \overline{\boldsymbol{\rho}}^{\lambda+1, t+1}\right)$ of oxygen and carbon dioxide. In our numerical approach, the reference density $\rho_{\text {ref }}$ and consumption rate $\bar{Q}$ (of each species $i$ ) for the next time step are evaluated at the end of each time step of the simulation. The reference density $\rho_{\text {ref }}$ is obtained using the macro-micro balance of mass condition $\left(\bar{\rho}=(1 / V) \int_{V} \rho \mathrm{d} V\right)$ and Equation (1). This definition is consistent with the condition that stored mass at macro-scale is same as the average micro-scale stored mass [15]. To further understand the micro-scale quantities that are needed to create the overall system of equations, the Jacobian matrix and force vector for a finite element $e$ with shape functions $N_{i}$ occupying a volume $\Omega^{\mathrm{e}}$ are expanded as follows:

$$
K_{i j}^{t+1, \lambda}=\left[\begin{array}{cc}
\frac{1}{\Delta t} \int_{\Omega^{\mathrm{e}}} N_{i} N_{j} \mathrm{~d} V-\int_{\Omega^{\mathrm{e}}} \frac{\mathrm{d} N_{i}}{\mathrm{~d} x_{p}} \bar{\kappa}_{p q}^{\mathrm{O}_{2} / \mathrm{O}_{2}} \frac{\mathrm{d} N_{j}}{\mathrm{~d} x_{q}} \mathrm{~d} V & -\int_{\Omega^{\mathrm{e}}} \frac{\mathrm{d} N_{i}}{\mathrm{~d} x_{p}} \bar{\kappa}_{p q}^{\mathrm{O}_{2} / \mathrm{CO}_{2}} \frac{\mathrm{d} N_{j}}{\mathrm{~d} x_{q}} \mathrm{~d} V \\
-\int_{\Omega^{\mathrm{e}}} \frac{\mathrm{d} N_{i}}{\mathrm{~d} x_{p}} \bar{\kappa}_{p q}^{\mathrm{CO}_{2} / \mathrm{O}_{2}} \frac{\mathrm{d} N_{j}}{\mathrm{~d} x_{q}} \mathrm{~d} V & \frac{1}{\Delta t} \int_{\Omega^{\mathrm{e}}} N_{i} N_{j} \mathrm{~d} V-\int_{\Omega^{\mathrm{e}}} \frac{\mathrm{d} N_{i}}{\mathrm{~d} x_{p}} \bar{\kappa}_{p q}^{\mathrm{CO}_{2} / \mathrm{CO}_{2}} \frac{\mathrm{d} N_{j}}{\mathrm{~d} x_{q}} \mathrm{~d} V
\end{array}\right]
$$




$$
f_{i}^{t+1, \lambda}=\left[\begin{array}{c}
-\int_{\Omega^{\mathrm{e}}} N_{i} \frac{\bar{\rho}_{\mathrm{O}_{2}}-\bar{\rho}_{\mathrm{O}_{2}}^{t}}{\Delta t} \mathrm{~d} V+\int_{\Omega^{\mathrm{e}}} \frac{\mathrm{d} N_{i}}{\mathrm{~d} x_{p}} \bar{q}_{O_{2} p} \mathrm{~d} V+\int_{\Omega^{\mathrm{e}}} N_{i} \bar{Q}_{\mathrm{O}_{2}}^{t} \mathrm{~d} V \\
-\int_{\Omega^{\mathrm{e}}} N_{i} \frac{\bar{\rho}_{\mathrm{CO}_{2}-\bar{\rho}_{\mathrm{CO}_{2}}^{t}} \mathrm{~d} V+\int_{\Omega^{\mathrm{e}}} \frac{\mathrm{d} N_{i}}{\mathrm{~d} x_{p}} \bar{q}_{C O_{2} p} \mathrm{~d} V-\int_{\Omega^{\mathrm{e}}} N_{i} \bar{Q}_{\mathrm{CO}_{2}}^{t} \mathrm{~d} V}{}
\end{array}\right]
$$

From the above equations, it is seen that homogenized diffusivities $\overline{\boldsymbol{\kappa}}^{A / B}$ relating the mass flux of species A with respect to the pressure gradient of species B need to be defined at each integration point in the macro-scale. The evaluation of the homogenized diffusivity for Taylor and Homogenization approaches are given as follows: In the Taylor model, the diffusivity is directly obtained by comparing Equations (10), (25) and (16) as:

$$
\overline{\boldsymbol{\kappa}}^{A / B}=\frac{1}{V} \int_{\Omega} \boldsymbol{\kappa}^{A / B} \mathrm{~d} V
$$

In the homogenization approach, the homogenized diffusivity can be obtained using a perturbation analysis [24] or direct matrix manipulation [15]. In the perturbation approach, components of the macroscopic density gradient is independently perturbed by a small amount $\varepsilon$ that affects the boundary conditions at the micro-scale through Equation (1). The micro-scale problem is solved again using the perturbed boundary conditions and the resulting perturbation in homogenized flux is used to compute the homogenized diffusivity. This involves solution of several additional microscale problems during each Newton-Raphson iteration at the macro-scale to compute the values involved in $\overline{\boldsymbol{\kappa}}^{A / B}$. In this work, we follow the FE matrix approach to obtain homogenized diffusivity by direct manipulation of the converged Jacobian and residual matrices of the micro-scale problem. The highlight of this approach is that the diffusivity can be obtained as a closed-form expression directly from the micro-scale solution. The steps to compute the homogenized diffusivity are as follows. In the finite element formulation (mesh with $N$ nodes), the unknown vector $\boldsymbol{\rho}$ and the normal flux vector $\boldsymbol{q}_{n}$ are given as:

$$
\{\rho\}=\left[\begin{array}{c}
\rho_{\mathrm{O}_{2}}^{1} \\
\rho_{\mathrm{CO}_{2}}^{1} \\
\vdots \\
\rho_{\mathrm{O}_{2}}^{N} \\
\rho_{\mathrm{CO}_{2}}^{N}
\end{array}\right] \text { and }\left\{q_{n}\right\}=\left[\begin{array}{c}
q_{\mathrm{O}_{2}}^{1} \\
q_{n_{\mathrm{CO}_{2}}}^{1} \\
\vdots \\
q_{\mathrm{O}_{2}}^{N} \\
q_{n_{\mathrm{CO}_{2}}}^{N}
\end{array}\right]
$$

Macroscopic flux is first written using the vector of normal fluxes on the external nodes of the microstructure $\left(\left\{q_{n}\right\}^{\text {ext }}\right)$ using finite element matrix representation as follows:

$$
\overline{\boldsymbol{q}}=\left[\begin{array}{c}
\overline{\boldsymbol{q}}_{\mathrm{O}_{2}} \\
\overline{\boldsymbol{q}}_{\mathrm{CO}_{2}}
\end{array}\right]=\frac{1}{V}\left[\begin{array}{l}
\int_{S} \boldsymbol{x} q_{\mathrm{O}_{2}} \mathrm{~d} S \\
\int_{S} \boldsymbol{x} q_{n_{\mathrm{CO}_{2}}} \mathrm{~d} S
\end{array}\right]=\boldsymbol{L}\left\{q_{n}\right\}^{\mathrm{ext}}
$$

where $L$ is a matrix of size $4 \times 2 N^{\text {ext }}$ where $N^{\text {ext }}$ is the number of nodes on the external boundary of the microstructure.

To compute the homogenized diffusivity ( $\overline{\boldsymbol{\kappa}}-\mathrm{a} 4 \times 4$ matrix), one needs to compute sensitivity of $\left\{q_{n}^{\text {ext }}\right\}$ to perturbations in the macroscopic density gradient $\delta(\overline{\nabla \rho})$ as:

$$
\delta \overline{\boldsymbol{q}}=\boldsymbol{L}\left\{\delta q_{n}\right\}^{\mathrm{ext}}=\overline{\boldsymbol{\kappa}}\left[\begin{array}{c}
\delta \bar{\nabla}_{\mathrm{O}_{2}} \\
\delta \overline{\nabla \rho}_{\mathrm{CO}_{2}}
\end{array}\right]=\overline{\boldsymbol{\kappa}}\{\delta(\overline{\nabla \rho})\}
$$


To obtain the homogenized diffusivity, the converged finite element solution from the NewtonRaphson iterations at the micro-scale is employed as follows:

$$
\left[\begin{array}{cc}
\boldsymbol{K}_{e e} & \boldsymbol{K}_{e i} \\
\boldsymbol{K}_{i e} & \boldsymbol{K}_{i i}
\end{array}\right]\left[\begin{array}{c}
\delta \boldsymbol{\rho}^{\mathrm{e}} \\
\delta \boldsymbol{\rho}^{\mathrm{i}}
\end{array}\right]=\left[\begin{array}{l}
0 \\
0
\end{array}\right]
$$

In the above equation, the assembled matrix $(\boldsymbol{K})$ on the left-hand side is the Jacobian matrix of the Newton-Raphson iteration. The residual on the right-hand side goes to zero since the micro-scale solution has converged. Vectors $\delta \boldsymbol{\rho}^{\mathrm{e}}$ and $\delta \boldsymbol{\rho}^{\mathrm{i}}$ contain the densities on the external and internal nodes of the microstructure, respectively. The submatrix $\boldsymbol{K}_{e e}$ contains entries in the Jacobian matrix corresponding to only the external nodes of the microstructure and is of size $2 N^{\mathrm{ext}} \times 2 N^{\mathrm{ext}}$. Using our boundary condition on the temperatures on the external nodes (Equation (1)), sensitivity of densities on external nodes of the micro-scale mesh to perturbation in the imposed macroscopic enthalpy gradient can be written as

$$
\left\{\delta \boldsymbol{\rho}^{\mathrm{e}}\right\}=\boldsymbol{G}\left[\begin{array}{c}
\delta \bar{\nabla}_{\mathrm{O}_{2}} \\
\delta \overline{\nabla \rho}_{\mathrm{CO}_{2}}
\end{array}\right]=\boldsymbol{G}\{\delta(\overline{\nabla \rho})\}
$$

where $G$ is a matrix of size $2 N^{\text {ext }} \times 4$.

Substituting the above relation into the converged matrix equation at the micro-scale and taking the known quantities to the right-hand side, we obtain the equation:

$$
\left[\begin{array}{cc}
\boldsymbol{K}_{e e} & \boldsymbol{K}_{e i} \\
\boldsymbol{K}_{i e} & \boldsymbol{K}_{i i}
\end{array}\right]\left[\begin{array}{c}
0 \\
\delta \boldsymbol{\rho}^{\mathrm{i}}
\end{array}\right]=\left[\begin{array}{c}
-\boldsymbol{K}_{e e} \boldsymbol{G}\{\delta(\overline{\nabla \rho})\} \\
-\boldsymbol{K}_{i e} \boldsymbol{G}\{\delta(\overline{\nabla \rho})\}
\end{array}\right]
$$

The vector on the right-hand side provides the sensitivity of microscopic flux to the macroscopic density gradient, which leads to the homogenized conductivity, $\overline{\boldsymbol{\kappa}}$, as follows:

$$
\begin{aligned}
\delta q_{n}{ }^{\mathrm{ext}} & =-\boldsymbol{K}_{e e} \boldsymbol{G}\{\delta(\overline{\nabla \rho})\} \\
\overline{\boldsymbol{\kappa}} & =-\boldsymbol{L} \boldsymbol{K}_{e e} \boldsymbol{G}
\end{aligned}
$$

To aid in speeding up the solution process for the multi-scale problem, the algorithm was parallelized using MPI. The macro-scale domain was decomposed and elements in each domain were distributed to different processors. The underlying micro-scale problems were solved in serial in each processor. The simulator was developed using object-oriented programming and was dynamically linked to the parallel toolbox PetSc [25] for parallel assembly and solution of linear systems. For solution of linear systems, a GMRES solver along with block Jacobi and ILU preconditioning from the PetSc toolbox was employed.

\section{TRACKING OF THE INTERFACE USING LEVEL SET METHOD AND ADAPTIVE MESHING}

Level set approach provides a natural way of tracking the degrading interface at the micro-scale during FE computations. To describe the interface, we construct a field $\phi$ such that at any time $t$, the interface is equal to the zero level set of $\phi$, i.e.

$$
S^{\mathrm{I}}(t)=\left\{x \in V^{+} \cup V^{-} \text {such that } \phi(x, t)=0\right\}
$$

Initially, $\phi$ is set equal to the signed distance function from the interface $S^{\mathrm{I}}$

$$
\phi(x, 0)= \begin{cases}+d(x, 0), & x \in V^{+} \\ 0, & x \in S^{\mathrm{I}} \\ -d(x, 0), & x \in V^{-}\end{cases}
$$


Table II. List of material constant for the carbon fiber oxidation example.

\begin{tabular}{lccc}
\hline Material constant & Matrix & Void & C-fiber \\
\hline Volumetric porosity & 0.1 & 1 & 0 \\
Permeability $\left(\frac{\mathrm{m}^{2}}{\mathrm{kPa}}\right)$ & $10^{-7}$ & 0 & 0 \\
Viscosity & 0.00018 & 1 & 0 \\
Areal porosity & & 32 & \\
$M_{\mathrm{O}_{2}}(\mathrm{~g} / \mathrm{mol})$ & 44 & \\
$M_{\mathrm{CO}}(\mathrm{g} / \mathrm{mol})$ & & 12 & \\
$M_{\mathrm{C}}(\mathrm{g} / \mathrm{mol})$ & & 6452.35 & \\
$k_{0}(\mathrm{~s}-1)$ & 118300 & \\
$E_{a}(\mathrm{~J} / \mathrm{mol})$ & & 1740 & \\
$\rho_{\mathrm{c}}\left(\mathrm{kg} / \mathrm{m}^{3}\right)$ & 3.5 & \\
$r_{0}(\mu \mathrm{m})$ & & &
\end{tabular}

where $d(\boldsymbol{x}, 0)$ is the normal distance of a point $\boldsymbol{x}$ from the interface. The idea behind the level set method is to move $\phi$ with the correct speed $\mathbf{v}$ at the interface. The interface position is thus implicitly stored in $\phi$. The equation of motion governing $\phi$ is given as follows [26]:

$$
\frac{\partial \phi}{\partial t}+v \cdot \nabla \phi=0
$$

This equation moves $\phi$ with correct speed at the interface so that $S^{\mathrm{I}}$ will always be equal to the zero level set of $\phi$. We employ the stabilized Galerkin formulation on unstructured adaptive grids for solving the level set equation. The numerical implementation of the level set method is same as that detailed in Reference [26].

The mass loss rate of oxygen has been calibrated as an Arrhenius equation using experimental results in [27]. To convert the measured mass loss rate to the velocity of oxidation at nodal points in the micro-scale oxidizing interface, we assume that the intact carbon fiber of initial radius $r_{0}$ oxidizes to a smaller radius $r$ after a short duration $\Delta t$. The velocity of recession at any point can then be found by scaling the mass loss rate with the initial as well as current radius $r_{0}$ and $r$ as below:

$$
\boldsymbol{v}=\frac{\mathrm{d} r}{\mathrm{~d} t} \boldsymbol{n}=\frac{r_{0}^{2}}{2 r} k_{0} \mathrm{e}^{\left(-E_{a} /(R T)\right)}\left(\frac{P_{\mathrm{O}_{2}}}{P_{\mathrm{O}_{2}}^{*}}\right)^{k_{1}} \boldsymbol{n}
$$

The experimentally calibrated parameters, $E_{a}, k_{0}, k_{1}$ from Reference [4], are listed in Table II. We approximate the current radius $r$ using the area of current carbon fiber $A$ as $r=\sqrt{A / \pi}$. Note that an inert coating (pyrolitic carbon) is generally applied on the carbon fiber to provide oxidation resistance. For simplicity, the ensuing simulations assume that the coating is not present and the carbon fiber is fully exposed to oxygen. Nevertheless, the effect of coatings can be easily included in this model by using a different recession velocity (Equation (38)) for the coating material.

\section{COMPUTATIONAL APPROACH AND NUMERICAL RESULTS}

The overall solution scheme is shown in Table III. Homogenization and the Taylor model differ in the way the micro-scale problem is solved, with the homogenization approach accounting for micro-scale equilibrium.

In this section, we focus on the problem of oxidation of an assembly of carbon fibers within a single carbon tow. Scanning electron micrographs of cross-section of carbon tows reported in References [28, 29] (Figure 3(b)) reveal arrangement of carbon fibers in a hexagonal lattice within a tow. Micrographs obtained at high magnifications show the oxidation of individual carbon fibers (Figure 3(c)) as well as the preferential oxidation of carbon fibers adjoining the porous matrix due to high oxygen availability. As the outer fibers oxidize, oxygen diffuses inward along the voids formed and reacts with the interior fibers. The oxidized carbon fiber front (Figure 3(b)) evolves 
Table III. Solution scheme for multi-scale modeling of carbon fiber oxidation.

(1) Initialize macro-scale model and assign a microstructure (and FE meshes) to every integration point.

(2) Apply time increment $\Delta t$ to the macro-scale problem.

(3) Iteration step:

(3.1) Assemble the macroscopic stiffness matrix.

(3.2) Solve the macroscopic system and compute density and the density gradient at each integration point.

(3.3) Loop over all integration points

(a) (Only done for the first NR iteration) Update the carbon fiber using level set method.

(b) Taylor model: Transfer densities to every point in the micro-scale mesh using Equation (10). Homogenization model: Transfer boundary conditions to micro-scale problem. Assemble and solve the micro-scale problem.

(c) Calculate the volume averaged macro-flux (Equation (16)), the source term (Equation (28)), the macro-diffusivity (Equation (29) for Taylor and Equation (34) for Homogenization models).

(3.4) Assemble the macroscopic residual vector.

(4) Check convergence, if not converged go to step 3, otherwise go to step 2.
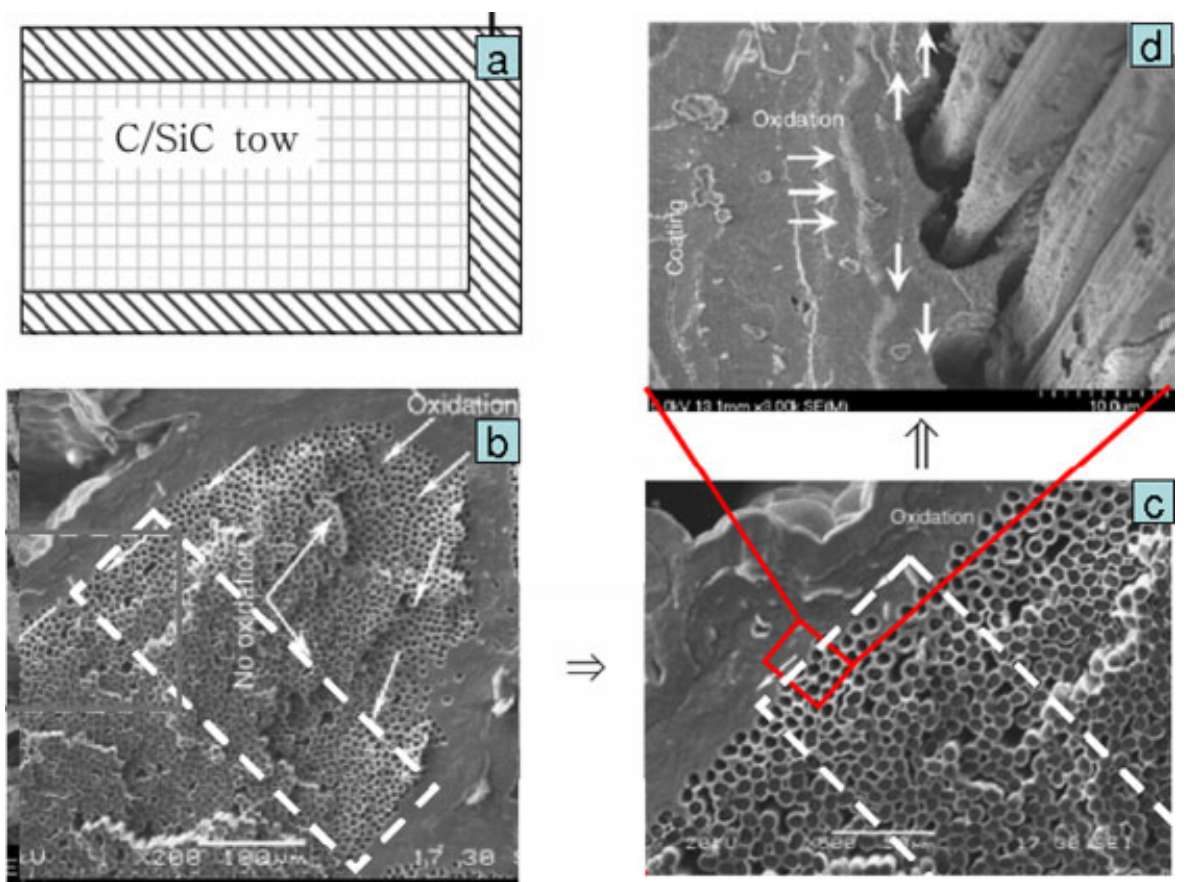

Figure 3. The micrographs represent experimental results of $\mathrm{C} / \mathrm{SiC}$ composite oxidation in a controlled environment reported in [28, 29]. Figure (d) shows the simulation cell: all the boundaries in the model are insulated except one side that is exposed to the external oxidizing environment. Size of the simulation cell at the macro-scale is indicated using dotted lines in (b). The simulation cell contains about 600 carbon fibers.

inward into the carbon fiber tow, until all the fibers are oxidized. In our numerical model, a section of carbon fiber tow (with about 600 carbon fibers) is taken to be our macroscopic model. The size of our macroscopic simulation cell (shown in Figure 4(a)) is indicated using dotted lines on the micrographs. In the macroscopic model, the evolution of homogenized carbon density is tracked as oxygen diffuses from the left end to the right end. All the boundaries in the model are insulated (as shown in Figure 3(a)) except one side that is exposed to the external environment of pure oxygen with imposed pressures of $P_{\mathrm{O}_{2}}=0.1 \mathrm{~atm}$ and $P_{\mathrm{CO}_{2}}=0$ atm for $t>0$. The system is modelled at a constant temperature of $950^{\circ} \mathrm{C}$. An initial condition (at $t=0$ ) of $P_{\mathrm{O}_{2}}=0.1 \mathrm{~atm}$ and $P_{\mathrm{CO}_{2}}=0 \mathrm{~atm}$ 


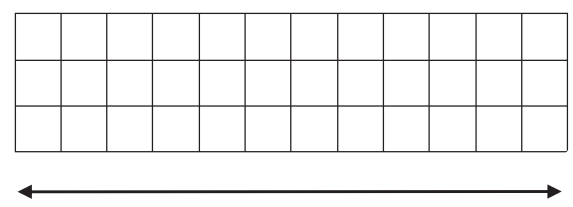

(a)

$0.4 \mathrm{~mm}$

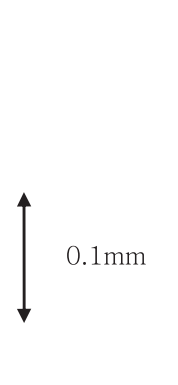

(b)

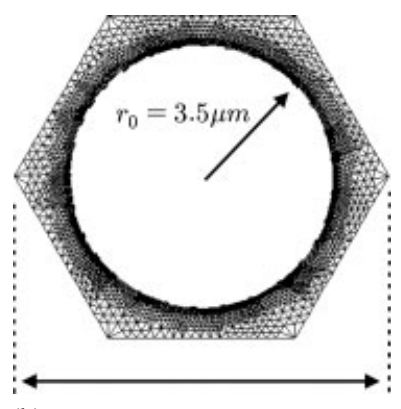

$10.0 \mu \mathrm{m}$

Figure 4. (a) Two-dimensional macro-scale finite element grid and (b) micro-scale finite element grid.
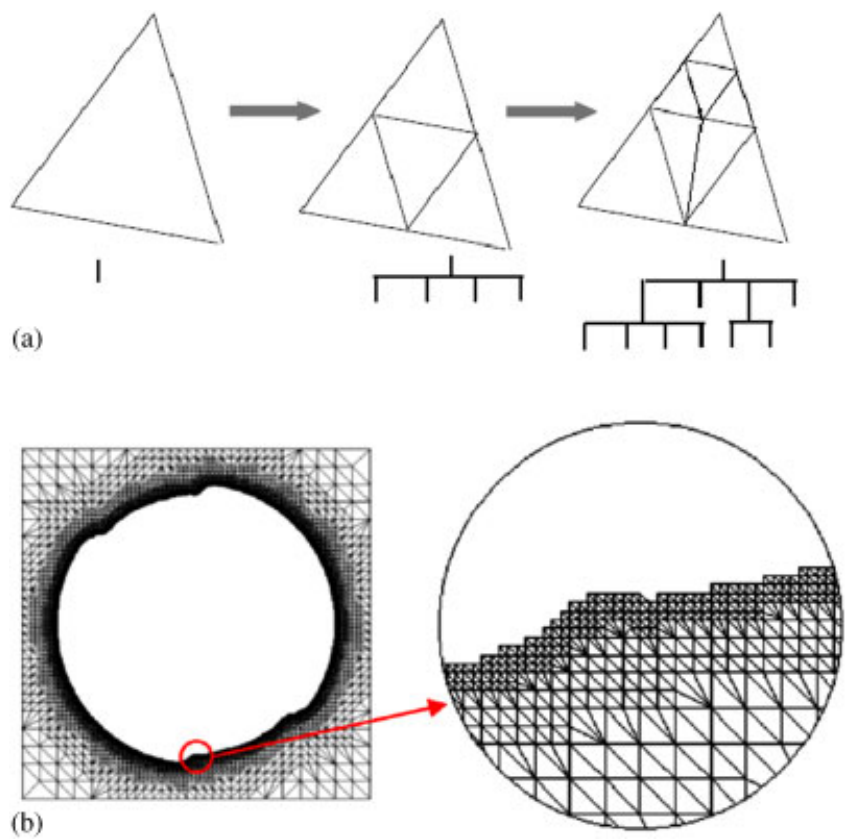

Figure 5. (a) A typical sequence of mesh refinement is shown along with the associated data structure and (b) oxidizing interface is accurately tracked using this adaptive meshing strategy.

is imposed at all points in the specimen. The micro-scale physics of carbon fiber oxidation is introduced by modeling a single carbon fiber within a hexagonal unit cell as shown in Figure 4(b). Since the carbon fibers do not mediate the diffusion of the gas species, the carbon fiber is not modeled in the micro-scale. The unit cell is assigned to each integration point in the macro-grid.

\subsection{Convergence study}

Micro-scale mesh convergence study was performed by considering the volume loss of carbon fibers as the convergence metric. Adaptive meshing is used to track the evolving micro-scale carbon fiber interface. A refinement procedure based on unstructured grids is employed in this work. During mesh refinement, each triangular element is subdivided into four triangles (as shown in Figure 5(a)) by adding middle nodes at its three edges. Information about the level of refinement is stored in a tree data structure following the approach in Reference [26], which is capable of tracking refined elements and ensures overall grid conformity. Owing to the use of such unstructured grids, we report the level of adaptivity using the smallest element edge length in the mesh. The adaptive meshing strategy used in the micro-scale is shown in Figure 5(b). The resulting mesh is coarser in the porous matrix and progressively becomes finer as we reach closer to the evolving interface. 

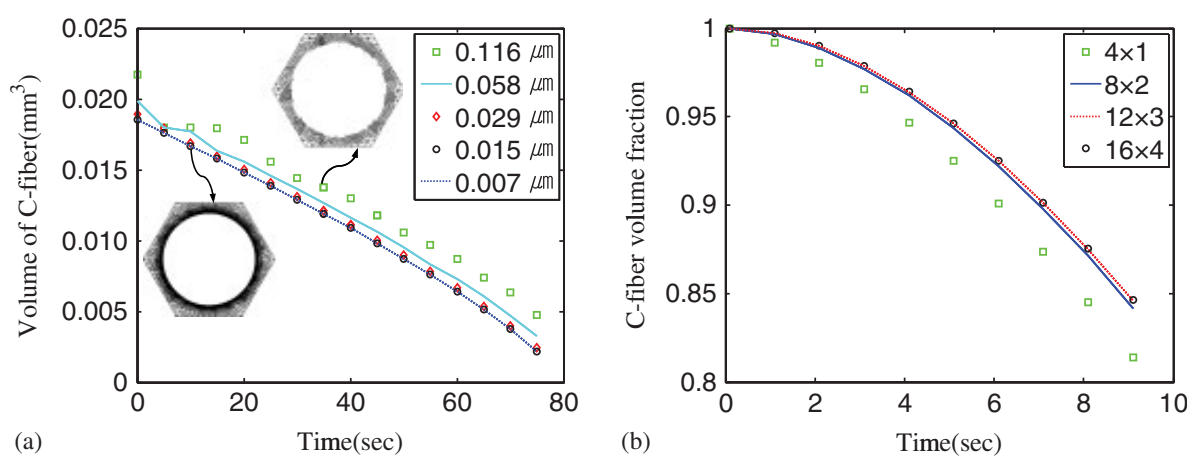

Figure 6. (a) Micro-scale convergence study: carbon fiber volume at the micro-scale is tracked as a function of time using different levels of adaptive meshing. The minimum element edge length in the mesh is reported here. The volume is reported assuming that the length in the $z$-direction is $1 \mathrm{~mm}$. (b) Macro-scale convergence study: carbon fiber volume fraction vs time is plotted for different mesh sizes. A mesh size of $a \times b$ indicates $a$ elements along the $x$-axis and $b$ elements along the $y$-axis.

For this micro-scale convergence study, a constant oxygen pressure of $0.05 \mathrm{~atm}$ is imposed at all points in the micro-scale unit cell. As shown in Figure 6(a), it is seen that the temporal evolution of carbon fiber volume converges as the level of mesh adaptivity increases. Based on this study, we selected the adaptive mesh setting that ensures a minimum element size of $0.029 \mu \mathrm{m}$ for the micro-scale problem. Subsequently, macro-scale grid convergence was tested using the configuration given in Figure 3(a)). An ambient pressure of $P_{\mathrm{O}_{2}}=0.1 \mathrm{~atm}$ and $P_{\mathrm{CO}_{2}}=0$ atm for $t>0$ is employed. The temporal evolution of macro-scale carbon fiber volume fraction is reported for various different macro-scale FE meshes in Figure 6(b). Taylor model was employed and timesteps were chosen adaptively during the simulation to ensure convergence of the overall multi-scale system. A grid with 12 elements along the $x$-axis and three elements along the $y$-axis was selected (a $12 \times 3$ grid, Figure 4(a)) based on this study and employed in the subsequent simulations. The numerical model is two dimensional and we have reported the volume changes assuming that the length in the $z$-direction is $1 \mathrm{~mm}$. To model oxygen flow along the fiber-matrix interface or cracks along the $z$-direction, three-dimensional models will be considered in the future.

\subsection{Numerical results of the Taylor and Homogenization multi-scale model}

Simulation of carbon tow oxidation in the configuration depicted in Figure 3(a) was carried out using both the Taylor and Homogenization approaches. In general, simulations reveal diffusioncontrolled kinetics at the beginning of oxidation. As the carbon fibers oxidize, a void is created in place of the oxidized carbon fiber. This greatly increases subsequent diffusion of oxygen toward the interior of the carbon tow. Owing to increased oxygen availability, a transition from diffusioncontrolled kinetics to reaction-controlled kinetics is seen. This is exemplified by a linear rate law, ie. oxidized carbon volume fraction increasing linearly with respect to time. A mixed rate law of the following form describes the overall oxidation process:

$$
\frac{\sqrt{x}}{k_{r}}+\frac{x}{k_{l}}=t
$$

where $k_{r}$ and $k_{l}$ are the rate constants. Figure 7(a) compares the oxidized carbon fiber volume fraction predicted at various times by the Taylor and homogenization approaches. Both Taylor and Homogenization approaches show similar behavior, and in both cases, the transition to a reaction-dominated regime occurs at a time of about $13 \mathrm{~s}$. However, the reaction rate constants predicted by the Taylor and Homogenization approaches are quite different as seen in Table IV. The Taylor model provides a overconstrained response compared with the FE homogenization approach due to strict imposition of oxygen and carbon dioxide densities within the unit cell. In fact, the Taylor model provides an upper-bound response for system, an effect well studied in literature (e.g. [13]). This is further exemplified in Figure 7(b), which shows the oxygen partial 

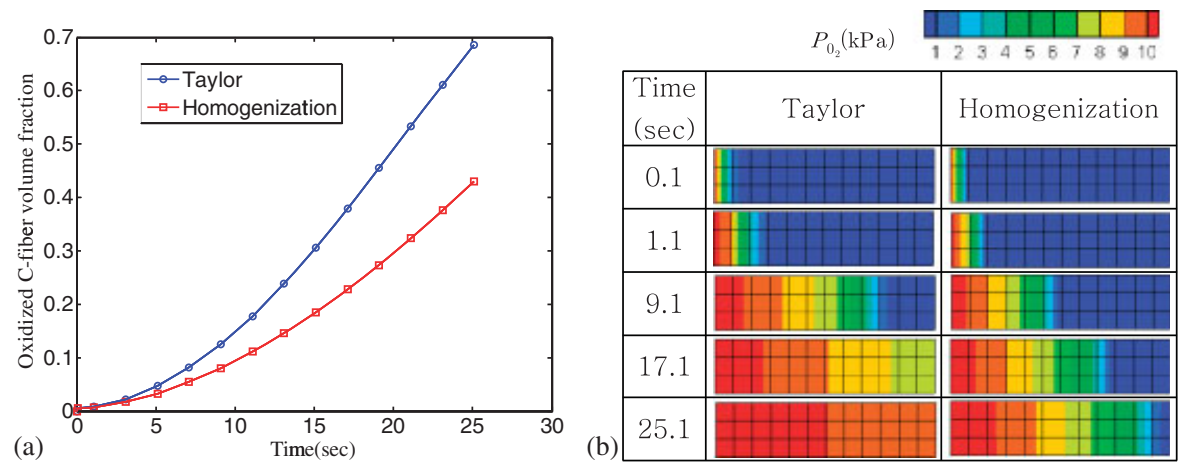

Figure 7. Comparison of the Taylor and Homogenization approaches: (a) recessed carbon fiber volume fraction vs time at the macro-scale and (b) oxygen pressure distributions at various times in the macro-scale.

Table IV. Square root and linear rate constants obtained by curve fitting the simulation results.

\begin{tabular}{lccc}
\hline & $k_{r} \times 10^{4}\left(1 / \mathrm{s}^{2}\right)$ & $k_{l} \times 10^{2}(1 / \mathrm{s})$ & Transition time (s) \\
\hline Taylor & 13.7 & 3.8 & 13.1 \\
Homogenization & 8.4 & 2.5 & 13.1 \\
\hline
\end{tabular}

pressure distribution within the macro-scale mesh at various times. The Taylor model, as expected, predicts faster oxygen transport within the tow compared with the FE homogenization approach. FE homogenization approach solves the micro-scale equilibrium equations and the micro-scale solution is more physically reasonable. However, the choice of selection of one model versus another is based on the relative importance of accuracy versus computational speed. In the Taylor model, there is no need for finite element computations at the micro-scale and the convergence of the overall non-linear multi-scale scheme is faster. In the case considered here, the Taylor model was solved four times faster than the homogenization model.

In order to illustrate changes in the carbon fiber configuration in the micro-scale, the Taylor model solution at selected six integration points is shown in Figure 8 at a time of $25.1 \mathrm{~s}$. Although the macro-problem studied corresponds to a one-dimensional diffusion, the micro-scale oxidation results shown in Figure 8 do not follow this trend. Indeed, the one-dimensional nature of the macroscopic density gradient is considered in the micro-scale simulation through the scale linking equation (Equation (1)). However, the characteristic length scale over which the macroscopic field variables vary is much larger than the size of the microscopic volume considered. Consequently, the contribution of the macroscopic gradient term to the micro-scale oxygen and carbon dioxide density (ie. term $\nabla \rho \cdot \boldsymbol{x}$ in Equation (1)) is not significant compared with the macroscopic oxygen density term (term $\rho_{\text {ref }}$ in Equation (1)). In other words, the macroscopic oxygen density field translates to a nearly constant oxygen density at the level of the RVE. This kind of uniform oxidation of carbon fibers is indeed seen from experimental micrographs as shown in Figure 3(d).

A comparison of the simulation results from the Taylor and homogenization approaches at various locations in the macroscopic mesh at a simulation time of $25.1 \mathrm{~s}$ is shown in Figure 9. In this figure, the tan-, white- and blue-colored areas indicate matrix, void and carbon fiber, respectively. Both the Taylor and homogenization models indicate complete oxidation of the carbon fibers located closest to the exposed macro-scale surface (left edge) at $25.1 \mathrm{~s}$. The oxygen partial pressure solution at the micro-scale shows the contrast between the two approaches. The boundary conditions in the Taylor model lead to enforcement of high oxygen densities within the matrix compared with the physically accurate solution from homogenization that satisfies micro-scale equilibrium. In the steady-state micro-scale solution, the oxygen density in the void (that is left behind after oxidation of the carbon fiber) is expected to be uniform due to much faster diffusion in 


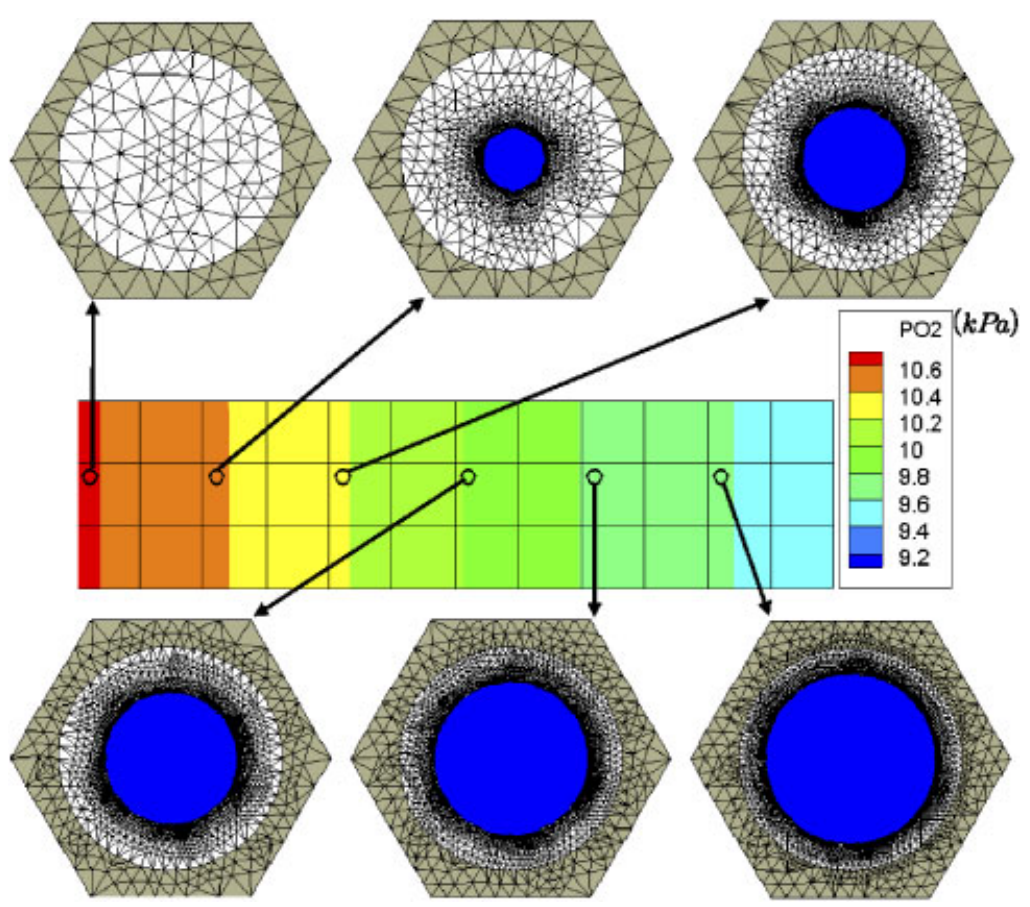

Figure 8. Carbon fiber configurations in micro at $25.1 \mathrm{~s}$ at $950^{\circ} \mathrm{C}$; tan-, white- and blue-colored areas indicate matrix, void and carbon fiber, respectively.

the void compared with the porous matrix. This is clearly seen from the homogenization approach, whereas in the Taylor model, a variation of oxygen density in the void is enforced. Further, in all cases, the Taylor model provides an upper bound for the oxygen density solution (and faster oxidation) at both macro- and micro-scales. The Taylor model predicts partial oxidation, whereas the homogenization approach predicts an almost intact carbon fiber at an integration point located on the right end. This effect is clearly seen by plotting the spatial distribution of carbon volume fraction in the composite at $0,11.1$ and $25 \mathrm{~s}$ (as shown in Figure 10).

The pore structure of $\mathrm{C} / \mathrm{SiC}$ composite is affected by thermally induced stresses in the component. Thermal stresses result in matrix cracks and de-bonds among the carbon fibers, the pyro-carbon coating and the $\mathrm{SiC}$ matrix. One would expect that the porosity in the matrix will be a complex function of temperature, heating rate and externally applied loads. Deformation and thermal shocks lead to increased damage in the matrix, thus increasing oxygen availability at degrading interfaces. Increased oxidation, in turn, leads to overall weakening of the material making it susceptible to further damage. This phenomena of strong 'stress-oxidation' coupling is explained in [30]. The micro-scale model presented here is a first step toward a multi-scale approach for studying stressoxidation coupling and associated mechanical property degradation in $\mathrm{C} / \mathrm{SiC}$ composites. In the future, we plan to model the structural loading and thermal cycling effects that lead to porosity evolution and increased oxygen diffusion in the matrix.

\section{CONCLUSION}

In this paper, a non-linear-coupled macro-micro finite element model is presented for addressing carbon fiber oxidation problems. Oxidation involves evolution of carbon fiber surfaces coupled with flux jump boundary conditions across interfaces that have not been addressed using the homogenization approaches. Homogenization of complex micro-scale behavior including moving interfaces and flux jumps has been performed in this work. Based on Hill's macro-homogeneity condition, macroscopic quantities are evaluated via consistent averaging of the microscopic values. 


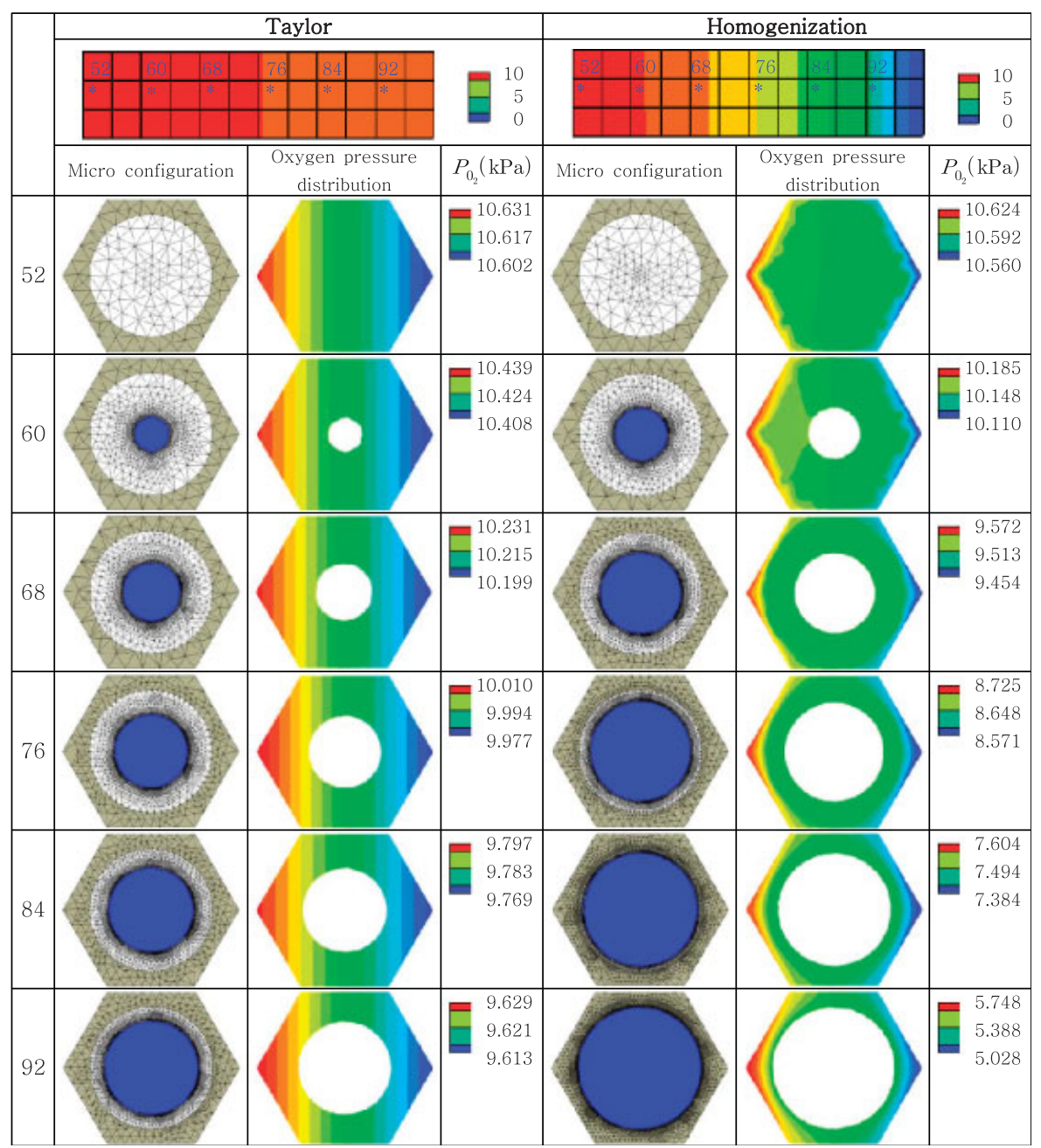

Figure 9. Comparison of carbon fiber configuration and micro-scale oxygen densities for the Taylor and homogenization approaches at a simulation time of $25.1 \mathrm{~s}$.
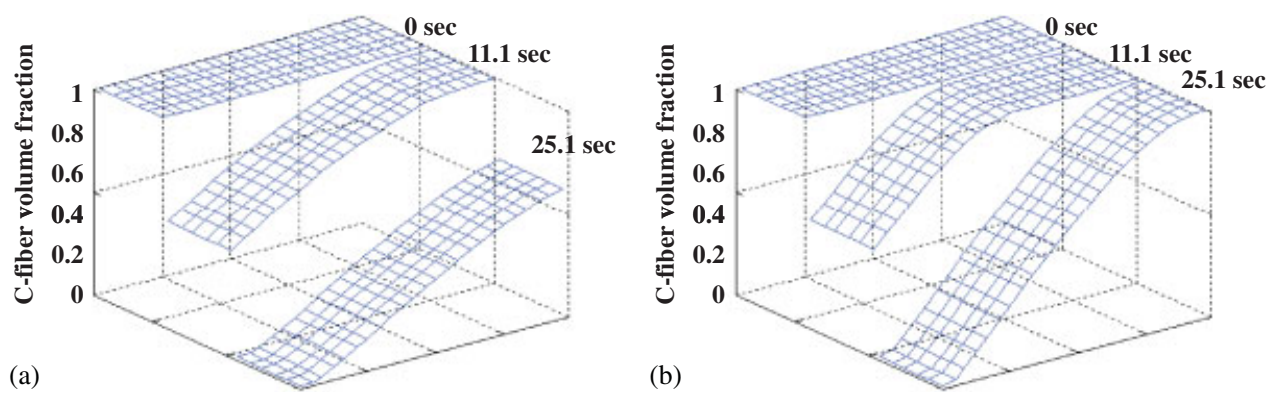

Figure 10. Spatial distributions of carbon fiber volume fraction at each integration points in macro at 0 , 11.1 and $25.1 \mathrm{~s}$ for (a) Taylor and (b) Homogenization model.

The micro-scale model incorporates the physics associated with oxidation including moving interfaces and flux discontinuities, whereas the macro-scale model needs to only model diffusion using continuous (homogenized) fields. Two multiscaling procedures were outlined in this work: one based on assumed solution in the micro-scale (Taylor model) and another based on finite element solution of the micro-scale problem (Homogenization approach). A nested finite element solution 
scheme is implemented into an FE framework and the macroscopic diffusivity is derived in a consistent manner for these two approaches.

The results from these two multi-scaling approaches were compared for a simple one-dimensional oxidation of a carbon fiber tow. In both models, the influence of microstructural evolution (e.g. carbon fiber oxidation) on the competition between macroscopic oxygen and carbon dioxide transport in the macro-scale can be introduced and treated effectively. The Taylor model is computationally efficient but provides an upper-bound response and predicts faster oxygen transport within the tow compared with the FE homogenization approach. The proposed method, when combined with thermo-mechanical field effects, constitutes a powerful tool, for modeling high-temperature oxidation of composites. The homogenization approach enhances the understanding and modeling of micro-scale interfacial phenomena and in the future, would allow modeling of interesting mechanisms such as interaction of porosity evolution with carbon fiber oxidation ('stress-oxidation coupling') that cannot be explained without incorporating microstructural details.

\section{APPENDIX A: HOMOGENIZED FLUX DERIVATION BASED ON HILL'S MACRO-HOMOGENEITY CONDITION}

The fluxes in the homogenization approach are derived such that the macro-homogeneity condition is satisfied. The micro-scale partial density field of each species $i$ is denoted as $\rho$ and no subscripts are used to maintain generality. Based on local mass balance equation (Equation (19)), it can be proved that the integral of normal mass flux over the unit cell surface tends to zero as follows:

$$
\begin{aligned}
\int_{S} q_{n} \mathrm{~d} S & =\int_{V} \nabla \cdot \boldsymbol{q} \mathrm{d} V-\int_{S^{\mathrm{I}}}\left[\left|q_{n}\right|\right] \mathrm{d} S^{\mathrm{I}} \\
& =-\int_{V} \nabla \cdot(\rho \boldsymbol{v}) \mathrm{d} V-\int_{S^{\mathrm{I}}}\left[\left|q_{n}\right|\right] \mathrm{d} S^{\mathrm{I}} \\
& =-\int_{S^{\mathrm{I}}}\left(v_{n}[|\rho|]+\left[\left|q_{n}\right|\right]\right) \mathrm{d} S^{\mathrm{I}}=0
\end{aligned}
$$

The above relation is subsequently used for homogenization of the micro-scale flux.

Application of the governing equation (Equation (18)) changes the macro-homogeneity condition to the following form:

$$
\begin{aligned}
\overline{\nabla \rho} \cdot \overline{\boldsymbol{q}}=\overline{\nabla \rho \cdot \boldsymbol{q}} & =\frac{1}{V} \int_{V}(\nabla \cdot(\rho \boldsymbol{q})-\rho \nabla \cdot \boldsymbol{q}) \mathrm{d} V \\
& =\frac{1}{V} \int_{S} \rho q_{n} \mathrm{~d} S+\frac{1}{V} \int_{S^{\mathrm{I}}}\left[\left|\rho q_{n}\right|\right] \mathrm{d} S^{\mathrm{I}}+\frac{1}{V} \int_{V} \rho \nabla \cdot(\rho \boldsymbol{v}) \mathrm{d} V
\end{aligned}
$$

We can reduce the first term in the above equation using the definition of micro-scale density (Equation (1)) and the homogeneous boundary conditions as:

$$
\begin{aligned}
\frac{1}{V} \int_{S} \rho q_{n} \mathrm{~d} S & =\frac{1}{V} \int_{S}\left[\rho_{\text {ref }}+\overline{\nabla \rho} \cdot \boldsymbol{x}\right] q_{n} \mathrm{~d} S \\
& =\overline{\nabla \rho} \cdot \frac{1}{V} \int_{S} \boldsymbol{x} q_{n} \mathrm{~d} S \quad\left(\text { using } \int_{S} q_{n} \mathrm{~d} S=0\right)
\end{aligned}
$$


The second and third terms in Equation (A3) are again reduced using the generalized divergence theorem as:

$$
\begin{aligned}
& \frac{1}{V} \int_{S^{\mathrm{I}}}\left[\left|\rho q_{n}\right|\right] \mathrm{d} S^{\mathrm{I}}+\frac{1}{V} \int_{V} \rho \nabla \cdot(\rho v) \mathrm{d} V \\
& \quad=\frac{1}{V} \int_{S^{\mathrm{I}}}\left[\left|\rho q_{n}\right|\right] \mathrm{d} S^{\mathrm{I}}+\frac{1}{V} \int_{V} \nabla \cdot\left(\rho^{2} v\right) \mathrm{d} V-\frac{1}{V} \int_{V} \nabla \rho \cdot(\rho v) \mathrm{d} V \\
& \quad=\frac{1}{V} \int_{S^{\mathrm{I}}}\left[\left|\rho q_{n}\right|\right] \mathrm{d} S^{\mathrm{I}}+\frac{1}{V} \int_{S^{\mathrm{I}}}\left[\left|\rho^{2}\right|\right] v_{n} \mathrm{~d} S^{\mathrm{I}}
\end{aligned}
$$

In the above derivation, we use local mass balance equation and the fact that the particle velocity is zero at all points in the material except the interface. The second term in the above equation contains the oxygen density field and flux jump across the oxidizing interface. This term is reduced as follows:

$$
\begin{aligned}
& \left(\left[\left|\rho q_{n}\right|\right]+\left[\left|\rho^{2}\right|\right] v_{n}\right)=\left(\rho_{+} q_{n+}-\rho_{-} q_{n-}\right)+\left(\rho_{+}^{2}-\rho_{-}^{2}\right) v_{n} \\
& \quad=\left(\rho_{+}+\rho_{-}\right)\left(v_{n}[|\rho|]+\left[\left|q_{n}\right|\right]\right)+\left(\rho_{+} q_{n-}-\rho_{-} q_{n+}\right) \\
& \quad=\left(\rho_{+} q_{n-}-\rho_{-} q_{n+}\right)=0 \text { on } S^{\mathrm{I}} \quad \text { since } \rho_{-}=0 \quad \text { and } q_{n-}=0 \text { on } S^{\mathrm{I}}
\end{aligned}
$$

In the above derivation, we have used the notion that oxygen density and oxygen flux is small inside the carbon fiber. Combining all the above equations, the macroscopic flux is obtained as:

$$
\overline{\boldsymbol{q}}=\frac{1}{V} \int_{S} \boldsymbol{x} q_{n} \mathrm{~d} S
$$

Using the governing equation at the micro-scale (Equation (18)), we obtain the expression for $\nabla \cdot \boldsymbol{x} \boldsymbol{q}$ as:

$$
\nabla \cdot \boldsymbol{x} \boldsymbol{q}=\boldsymbol{q}+\boldsymbol{x} \nabla \cdot \boldsymbol{q}=\boldsymbol{q}-\boldsymbol{x} \nabla \cdot(\rho \boldsymbol{v})
$$

Using the above equation and application of the generalized divergence theorem, we can obtain the volume average of heat flux as:

$$
\begin{aligned}
\frac{1}{V} \int \boldsymbol{q} \mathrm{d} V & =\frac{1}{V} \int_{V}(\nabla \cdot \boldsymbol{x} \boldsymbol{q}+\boldsymbol{x} \nabla \cdot(\rho \boldsymbol{v})) \mathrm{d} V \\
& =\frac{1}{V} \int_{S} \boldsymbol{x} q_{n} \mathrm{~d} S+\frac{1}{V} \int_{S^{\mathrm{I}}} \boldsymbol{x}\left[\left|q_{n}\right|\right] \mathrm{d} S^{\mathrm{I}}+\frac{1}{V} \int_{V} \boldsymbol{x} \nabla \cdot(\rho \boldsymbol{v}) \mathrm{d} V
\end{aligned}
$$

The last term in the above equation can be rewritten as:

$$
\frac{1}{V} \int_{V} \boldsymbol{x} \nabla \cdot(\rho \boldsymbol{v}) \mathrm{d} V=\frac{1}{V} \int_{V} \nabla \cdot(\rho \boldsymbol{x} \otimes \boldsymbol{v}) \mathrm{d} V-\frac{1}{V} \int_{V} \rho \boldsymbol{v} \mathrm{d} V=\frac{1}{V} \int_{S^{1}} \boldsymbol{x}[[\rho]] v_{n} \mathrm{~d} S^{\mathrm{I}}
$$

In the above derivation, we have used the fact that particle velocity is zero at all points in the domain except at the interface to eliminate the terms involving volume integral of velocity. The above equation can be used along with the interface mass balance condition (Equation (19)) to 
obtain the expression for volume average of heat flux as:

$$
\frac{1}{V} \int \boldsymbol{q} \mathrm{d} V=\frac{1}{V} \int_{S} \boldsymbol{x} q_{n} \mathrm{~d} S+\frac{1}{V} \int_{S^{\mathrm{I}}} \boldsymbol{x}\left(\left[\left|q_{n}\right|\right]+[[\rho]] v_{n}\right) \mathrm{d} S^{\mathrm{I}}=\frac{1}{V} \int_{S} \boldsymbol{x} q_{n} \mathrm{~d} S=\overline{\boldsymbol{q}}
$$

As a consequence of this derivation, we prove that the micro-scale volume average flux is equal to the macro-scale flux as a consequence of Hill's macro-homogeneity condition.

\section{ACKNOWLEDGEMENTS}

This work was sponsored by NASA Constellation University Institutes Project under grant NCC3-989 with Claudia Meyer as the project manager.

\section{REFERENCES}

1. Glass DE. Ceramic matrix composite (CMC) thermal protection systems (TPS) and hot structures for hypersonic vehicles. Fifteenth AIAA Space Planes and Hypersonic Systems and Technologies Conference, Dayton, Ohio, AIAA-paper 2008-2682, 28 April-1 May 2008.

2. Eckel AJ, Cawley JD, Parthasarathy TA. Oxidation kinetics of a continuous carbon phase in a nonreactive matrix. Journal of the American Ceramic Society 1995; 78(4):972-980.

3. Halbig MC. The oxidation kinetics of continuous carbon fibers in a cracked ceramic matrix composite. NASA/TM2001-210520, 2001.

4. Sullivan RM. A model for the oxidation of carbon silicon carbide composite structures. Carbon 2005; 43(2): 275-285.

5. Hashin Z. Analysis of composite materials. Journal of Applied Mechanics 1983; 50:481-505.

6. Rosen BW, Hashin Z. Effective thermal expansion coefficients and specific heats of composite materials. International Journal of Engineering Science 1970; 8:157-173.

7. Noor AK, Shah RS. Effective thermoelastic and thermal properties of unidirectional fiber-reinforced composites and their sensitivity coefficients. Composite Structures 1993; 26:7-23.

8. Auriault JL. Effective macroscopic description of heat conduction in periodic composites. International Journal of Heat and Mass Transfer 1983; 26(6):861-869.

9. Guedes JM, Kikuchi N. Preprocessing and postprocessing for materials based on the homogenization method with adaptive finite element methods. Computer Methods in Applied Mechanics and Engineering 1990; 83:143-198.

10. Jiang M, Jasiuk I, Ostoja-Starzewski M. Apparent thermal conductivity of periodic two-dimensional composites. Computational Materials Science 2002; 25:329-338.

11. Smit RJM, Brekelmans WAM, Meijer HEH. Prediction of the mechanical behaviour of nonlinear heterogeneous systems by multi-level finite element modeling. Computer Methods in Applied Mechanics and Engineering 1998; 155:181-192.

12. Miehe C, Schroder J, Schotte J. Computational homogenization analysis in finite plasticity. Simulation of texture development in polycrystalline materials. Computer Methods in Applied Mechanics and Engineering 1999; 171:387-418.

13. Sundararaghavan V, Zabaras N. Design of microstructure-sensitive properties in elasto-viscoplastic polycrystals using multi-scale homogenization. International Journal of Plasticity 2006; 22:1799-1824.

14. Belytschko T, Loehnert S, Song JH. Multiscale aggregating discontinuities: a method for circumventing loss of material stability. International Journal for Numerical Methods in Engineering 2008; 73:869-894.

15. Lee S, Sundararaghavan V. Multiscale modeling of moving interface problems with flux jumps: application to solidification. Computational Mechanics 2009; 44(3):297-307.

16. Ozdemir I, Brekelmans WAM, Geers MGD. Computational homogenization for heat conduction in heterogeneous solids. International Journal for Numerical Methods in Engineering 2008; 73:185-204.

17. Hill R. On constitutive macro-variables for heterogeneous solids at finite strain. Proceedings of the Royal Society of London A 1972; 326:131-147.

18. Ward JT, Hamblen JB. Influence of diffusion of oxygen on the rate of combustion of solid carbon. Industrial and Engineering Chemistry 1927; 19(9):1025-1027.

19. Ostoja-Starzewski M. Towards stochastic continuum thermodynamics. Journal of Non-equilibrium Thermodynamics 2002; 27:335-348.

20. Rao VS, Hughes TJR, Garikipati K. On modelling thermal oxidation of Silicon II: numerical aspects. International Journal for Numerical Methods in Engineering 2000; 47:359-377.

21. LeVeque R. Numerical Methods for Conservation, aws. Birkhauser: Basel, 1992.

22. Bacos MP, Dorvaux JM, Lavigne O, Talandier J. C/C composite oxidation model III. Physical basis, limitations and applications. Carbon 2000; 38(1):105-117.

23. Bird RB, Stewart WE, Lightfoot EN. Transport Phenomena. Wiley: New York, NY, 1960.

24. Miehe C. Numerical computation of algorithmic (consistent) tangent moduli in large-strain computational inelasticity. Computer Methods in Applied Mechanics and Engineering 1996; 134(3-4):223-240. 
25. Balay S, Buschelman K, Eijkhout V, Gropp WD, Kaushik D, Knepley MG, McInnes LC, Smith BF, Zhang H. PETSc Users Manual ANL-95/11, revision 2.1.5. Argonne National Laboratory, 2004.

26. Tan L, Zabaras N. A level set simulation of dendritic solidification of multi-component alloys. Journal of Computational Physics 2007; 221(1):9-40.

27. Halbig MC. Carbon oxidation studies and the evaluation of oxidation inhibited C/SiC composites. Twenty-Eighth Annual Conference on Composites, Materials and Structures, Cocoa Beach, FL, U.S.A. United States Advanced Ceramics Association, 2004.

28. Mei H, Cheng L, Zhang L. Damage mechanisms of $\mathrm{C} / \mathrm{SiC}$ composites subjected to constant load and thermal cycling in oxidizing atmosphere. Scripta Materialia 2006; 54:163-168.

29. Mei H, Cheng L, Zhang L. Thermal cycling damage mechanisms of $\mathrm{C} / \mathrm{SiC}$ composites in displacement constraint and oxidizing atmosphere. Journal of the American Ceramic Society 2006; 89(7):2330-2334.

30. Verrilli M, Calomino A. Temperature dependence on the strength and stress rupture behavior of a carbon fiber-reinforced silicon carbide matrix (C/SiC) composite. Ceramic Engineering and Science Proceedings 2003; 24(4):443-449. 